\title{
Simultaneous Presentation of Lymphomatoid Granulomatosis and Multiple myeloma in an Immunodeficient Patient with Rheumatoid Arthritis
}

\author{
Shin Lee ${ }^{1}$, Eiju Negoro ${ }^{1}$, Hisashi Oki ${ }^{2}$, Yoshiaki Imamura ${ }^{3}$ and Takahiro Yamauchi ${ }^{1}$
}

\begin{abstract}
:
A 75-year-old Japanese woman with a 20-year history of rheumatoid arthritis presented with symptomatic bilateral pleural effusion and lung and brain tumors. She had received methotrexate for five years and tacrolimus for one year. A brain biopsy specimen showed the pathological features of lymphoproliferative disease, but a bone marrow biopsy showed proliferation of plasma cells. She was finally diagnosed with coexistent lymphomatoid granulomatosis (LYG) of the brain and lung and multiple myeloma (MM) of the bone marrow and received chemotherapy for both. This report shows that immunodeficient patients are at risk of developing the unusual coexistence of LYG and MM.
\end{abstract}

Key words: lymphomatoid granulomatosis, multiple myeloma, rheumatoid arthritis, methotrexate, tacrolimus

(Intern Med 58: 2845-2849, 2019)

(DOI: 10.2169/internalmedicine.2811-19)

\section{Introduction}

Continuous use of immunosuppressive agents such as methotrexate (MTX) and tacrolimus (TAC) sometimes induces hematological malignancies including lymphoproliferative disease (LPD) (1-3). Lymphomatoid granulomatosis (LYG), a rare type of LPD, can also be induced by longterm immunosuppression (4-6). Although LPD sometimes appears concurrently with other hematological disorders, few cases of the coexistence of LPD and multiple myeloma (MM) have been described $(7,8)$. To our knowledge, the simultaneous presentation of LYG and MM has not been reported previously.

In this report, the first case of the coexistence of LYG and MM induced by MTX and TAC is presented. Physicians should keep in mind that there is a risk of developing multiple hematological malignancies in patients on long-term immunosuppressive therapy, as in the present patient.

\section{Case Report}

A 75-year-old Japanese woman with a 20-year history of rheumatoid arthritis (RA) presented with dyspnea and temporary seizures. The patient had received MTX for 5 years (4 mg per week for 6 months, $6 \mathrm{mg}$ per week for 6 months, and $8 \mathrm{mg}$ per week for 4 years) and $1 \mathrm{mg}$ per day of TAC for 1 year for RA.

Her height was $146 \mathrm{~cm}$, weight $47.9 \mathrm{~kg}$, temperature $37.0^{\circ} \mathrm{C}$, heart rate 111 beats per minute, blood pressure 135/ $92 \mathrm{mmHg}$, and respiratory rate 20 breaths per minute. The patient had an oxygen saturation of $96 \%$ on $2 \mathrm{~L} /$ minute oxygenation through nasal cannulae on admission. Her Eastern Cooperative Oncology Group performance status (PS) was 4 due to severe arthritis and dyspnea caused by bilateral pulmonary effusion (PE). Her clinical laboratory data on admission are shown in Table.

Serum immunoelectrophoresis detected $\operatorname{IgA}-\lambda$ M-protein, urine protein electrophoresis detected Bence Jones protein, the $\kappa / \lambda$ ratio of serum immunoglobulin-free light chain was

\footnotetext{
${ }^{1}$ Department of Hematology and Oncology, Faculty of Medical Sciences, University of Fukui, Japan, ${ }^{2}$ Department of Orthopaedics and Rehabilitation Medicine, Faculty of Medical Sciences, University of Fukui, Japan and ${ }^{3}$ Division of Surgical Pathology, University of Fukui Hospital, Japan

Received: February 7, 2019; Accepted: April 11, 2019; Advance Publication by J-STAGE: June 27, 2019

Correspondence to Dr. Shin Lee, leesin.581020@gmail.com
} 
Table. Blood Chemistry Test Results on Admission.

\begin{tabular}{lclc}
\hline Hematology & & Biochemistry & \\
White blood cell count & $9,400 / \mu \mathrm{L}$ & Total protein & $5.8 \mathrm{~g} / \mathrm{dL}$ \\
Lymphocyte count & $1,494 / \mu \mathrm{L}$ & Albumin & $3.1 \mathrm{~g} / \mathrm{dL}$ \\
Red blood cell & $332 \times 10^{4} / \mu \mathrm{L}$ & Total bilirubin & $0.3 \mathrm{mg} / \mathrm{dL}$ \\
Hemoglobin & $10.2 \mathrm{~g} / \mathrm{dL}$ & AST & $19 \mathrm{IU} / \mathrm{L}$ \\
Hematocrit & $33.4 \%$ & ALT & $8 \mathrm{IU} / \mathrm{L}$ \\
Platelets & $28.7 \times 10^{4} / \mu \mathrm{L}$ & Lactate dehydrogenase & $359 \mathrm{IU} / \mathrm{L}$ \\
& & Blood urea nitrogen & $16 \mathrm{mg} / \mathrm{dL}$ \\
Immunology & & Creatinine & $0.42 \mathrm{mg} / \mathrm{dL}$ \\
IgA & $1,048 \mathrm{mg} / \mathrm{dL}$ & C-reactive protein & $0.74 \mathrm{mg} / \mathrm{dL}$ \\
IgG & $422 \mathrm{mg} / \mathrm{dL}$ & $\beta 2-$-microglobulin & $3.2 \mathrm{mg} / \mathrm{dL}$ \\
IgM & $17.6 \mathrm{mg} / \mathrm{dL}$ & sIL-2R & $764 \mathrm{IU} / \mathrm{L}$ \\
\hline
\end{tabular}

ALT: alanine aminotransferase, AST: aspartate aminotransferase, IgA: immunoglobulin A, IgG: immunoglobulin G, IgM: immunoglobulin M, sIL-2R: soluble interleukin-2 receptor
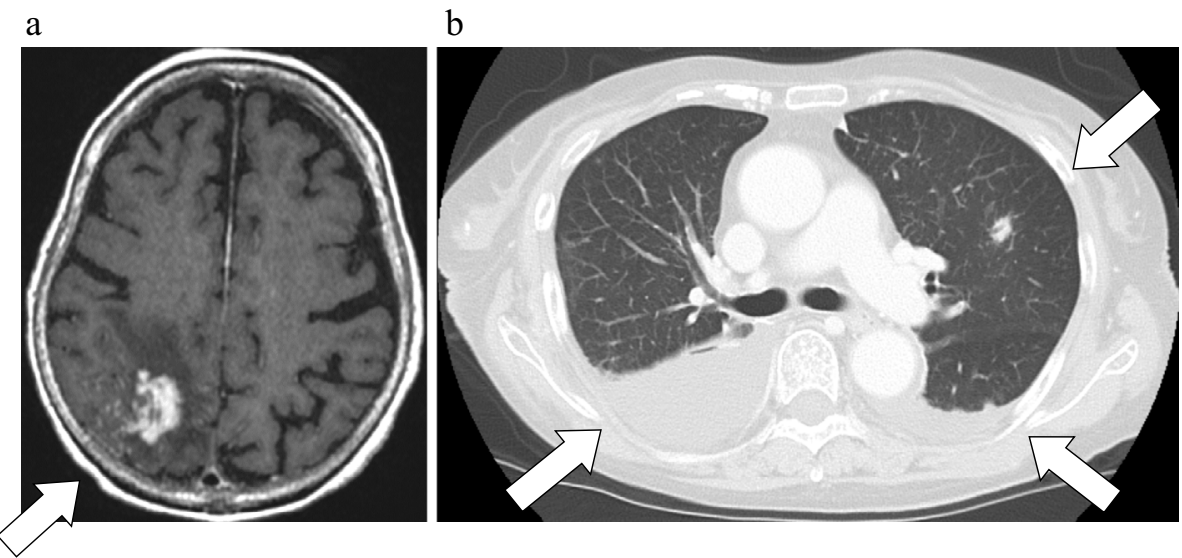

Figure 1. Brain gadolinium-enhanced magnetic resonance (Ga-MRI) and contrast-enhanced computed tomography (CECT) images at the onset. a: Brain Ga-MRI imaging at the onset. The arrow indicates enhancement of the poorly marginated lesions in the right parietal lobe. b: CECT of the chest and abdomen at the onset. The arrows indicate a poorly defined nodule in the left upper lobe and bilateral pleural effusion.

0.026, and Epstein-Barr virus (EBV) DNA was not detected in serum. Brain gadolinium-enhanced magnetic resonance imaging showed the enhancement of poorly marginated lesions in the right parietal lobe and left occipital lobe (Fig. 1a). Chest and abdominal contrast-enhanced computed tomography showed a poorly defined nodule in the left upper lobe and bilateral PEs (Fig. 1b). MTX and TAC were discontinued due to the possibility of immunosuppressive agent-induced LPD, but there was no improvement in the brain or lung tumors or bilateral PE. The pleural fluid showed high TP $(5.8 \mathrm{~g} / \mathrm{dL})$ and high lactate dehydrogenase (LDH) (359 IU/L) concentrations, but no malignant cells were detected in the pathological cytology or flow cytometric analysis. A biopsy of the brain tumor in the right parietal lobe, performed 11 days after the discontinuation of MTX showed the pathological features of LPD (Fig. 2a and b). In contrast, plasma cells in the bone marrow aspiration specimen were increased $(18.8 \%)$, and there was no evidence of LPD involvement in the bone marrow biopsy specimen. A flow cytometry analysis of the bone marrow revealed the expression of CD38 and CD138 and a discrepancy with immunoglobulin-free light chain $(\kappa<\lambda)$, with the absence of CD19 and CD56. A fluorescence in situ hybridization analysis of a bone marrow specimen was negative for $\operatorname{IgH}-$ FGFR3 fusion, p53 mutation, IgH-CCND1 fusion, and IgHMAF fusion. EBV-encoded small RNA-1 in situ hybridization (EBER-ISH) staining of these abnormal plasma cells showed negative results. Taken together, these findings led to the diagnosis of MM in the bone marrow (Fig. 2c), which was incompatible with the pathological findings of the brain biopsy.

Five weeks after the pathological consultation, the patient was finally diagnosed with coexistent EBV-associated LYG (grade 2) in the brain biopsy specimens and MM in the bone marrow clot and biopsy specimens. Hematoxylin and Eosin staining showed scattered perivascular infiltrates of medium-sized and large atypical lymphocytes (Fig. 2a and b). Results of Immunostaining using the CD20 

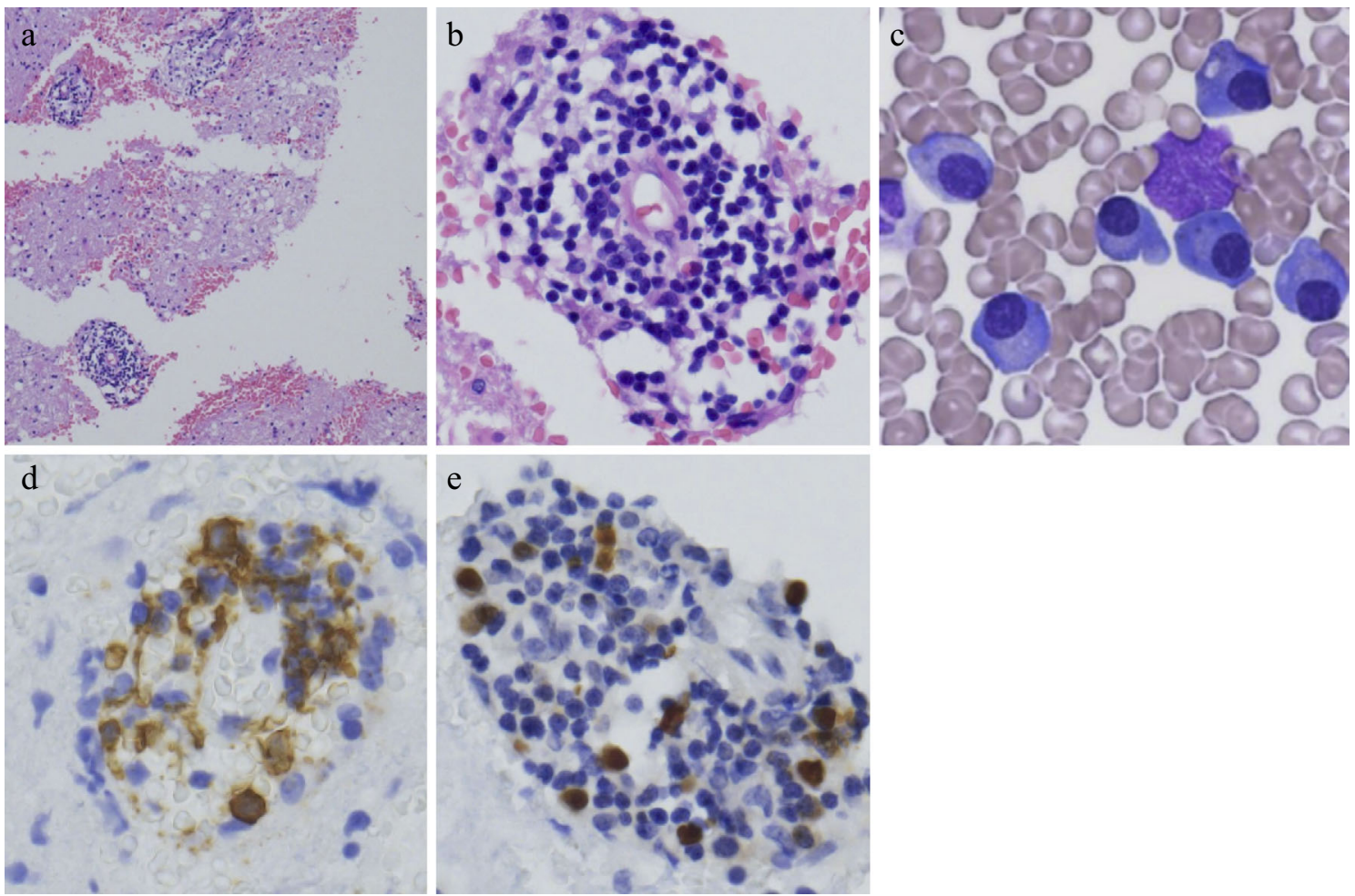

Figure 2. Pathological findings of a brain biopsy specimen and bone marrow aspiration specimen. a, b: Hematoxylin and Eosin staining of a brain biopsy specimen shows the scattered perivascular infiltrates of medium-sized to large atypical lymphocytes $(a, \times 10$ magnification; $b, \times 60$ magnification). c: May-Giemsa staining of the bone marrow aspiration specimen shows the proliferation of plasma cells (18.8\%), but no EBER-positive cells are seen. d: Immunostaining using the CD20 antibody ( $\times 60$ magnification) of a brain biopsy specimen. e: Immunostaining using EBV-encoded small RNA-1 in situ hybridization ( $\times 60$ magnification) of a brain biopsy specimen.

a

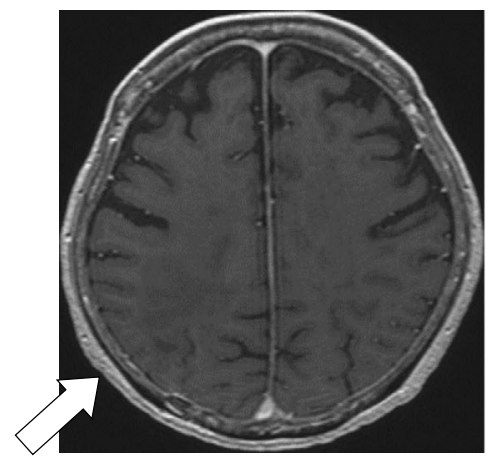

b

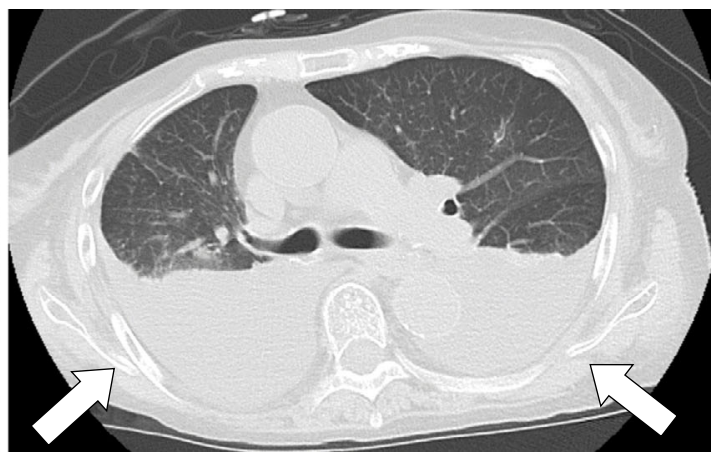

Figure 3. Repeated brain G-MRI and CECT images one month after starting oral prednisolone (PSL) followed by a brain biopsy. a: Repeated brain Ga-MRI images one month after starting oral PSL followed by a brain biopsy. The arrow indicates that oral PSL remarkably reduced the volume of brain enhancement in the right parietal lobe. b: Repeated CECT images of the chest and abdomen one month after starting oral PSL followed by a brain biopsy. The arrows indicate that oral PSL remarkably reduced the volume of the lung nodule in the left upper lobe. However, the bilateral pleural effusion had progressed despite oral PSL therapy.

antibody and EBER-ISH are shown in Fig. 2d and e. The CD20 and EBER-ISH-positive atypical B lymphocytes indicated EBV-induced B lymphocyte transformation.

A brain biopsy performed after 1 month of administration of oral prednisolone (PSL; $10 \mathrm{mg} /$ day) showed markedly re- duced brain and lung tumor volumes (Fig. 3a) but progression of the bilateral PE (Fig. 3b). The patient developed normocytic anemia $(\mathrm{Hb} 9.0 \mathrm{~g} / \mathrm{dL}$, mean corpuscular volume 98 $\mathrm{fL}$, reticulocytes $0.046 \times 10^{6} / \mu \mathrm{L}$ ), which was thought to have been caused by MM after the exclusion of hemolytic ane- 
mia. The patient underwent treatment for symptomatic MM with Ld therapy (cycles every 4 weeks of lenalidomide 15 $\mathrm{mg} / \mathrm{body}$ on days 1 to 21 and dexamethasone $40 \mathrm{mg} / \mathrm{body}$ on days $1,8,15$, and 22). Two cycles of Ld therapy slightly reduced the bilateral $\mathrm{PE}$ and led to a partial response (PR) of the MM. After the final diagnosis of LYG, the patient underwent 2 cycles of reduced R-CHOP (cycles every 3 weeks of rituximab $375 \mathrm{mg} / \mathrm{m}^{2}$ on day 1 , cyclophosphamide 375 $\mathrm{mg} / \mathrm{m}^{2}$ on day 1 , doxorubicin $25 \mathrm{mg} / \mathrm{m}^{2}$ on day 1 , vincristine $0.7 \mathrm{mg} / \mathrm{m}^{2}$ on day 1 , and PSL $100 \mathrm{mg} /$ body on days 1 to 5 ), and this therapy induced a clear reduction in the bilateral PE and led to PR of the LYG.

However, the treatment was discontinued because of the patient's and her family's refusal to continue due to severe febrile neutropenia, malaise, and anorexia. The patient ultimately died of LYG in the brain and lung with bilateral PE one month after recurrence and nine months after the onset.

\section{Discussion}

To our knowledge, this is the first reported case of the simultaneous presentation of LYG and MM induced by the long-term use of immunosuppressive agents including MTX.

A previous study reported that the incidence of hematological malignancies after drug exposure in RA was $0.39 \%$, most of which were LPD (55.9\%), leukemia (28.8\%), and MM (15.3\%), and they were reported to be frequently associated with EBV (1). A previous study showed that latent EBV-infected B cells could escape from host cytotoxic T lymphocytes under immunodeficient conditions, which might lead to the development of hematological malignancies (9). EBV-DNA in blood is a prognostic biomarker of EBV-associated LPD and has good concordance with EBER-ISH, but it also has an association with the tumor burden (10). In the present case, the discrepancy between the EBER-ISH findings and EBV-DNA in blood might have been due to a low tumor burden. LYG cells in the brain were positive on EBER-ISH staining, but no evidence of EBV infection was detected in the abnormal plasma cells in the bone marrow, possibly indicating that the LYG and MM in the present patient arose from different origins, although the significance of this association remains unclear.

Thus far, few cases of LPD and MM appearing either simultaneously or sequentially have been reported $(8,11,12)$. The treatment of LPD is sometimes prioritized over that of MM when LPD presents with a more aggressive clinical behavior than the co-existent MM (12). LYG is a rare category of LPD and has been classified as grade 1 to 3 based on the proportion of EBV-positive B lymphocytes present (13). Although several patients without symptoms and with histological low-grade (1 and 2) disease have achieved spontaneous remission (14), the majority have shown a poor prognosis with a high mortality (more than 50\%) and short median survival time (14 months) (15). Patients with a high histological grade (3), any symptoms, and extensive disease, such as that with neurologic involvement, should be treated as having diffuse large B-cell lymphoma and administrated multi-agent chemotherapy, given the poor prognosis $(14,15)$. While the spontaneous regression of LYG after discontinuation of MTX and TAC was expected, Ld therapy was started first for symptomatic MM in the present case. However, the LYG persisted despite the discontinuation of immunosuppressive agents and administration of chemotherapy for the coexistent MM. Of note, the brain involvement of LYG was an adverse prognostic factor that further justified the administration of chemotherapy. The brain involvement and poor PS probably resulted in the early relapse of LYG, which was thought to have influenced the prognosis in the present case.

We reported an unusual case of the simultaneous presentation of EBV-associated LYG and MM induced by the immunosuppressive agents, MTX and TAC. Physicians should be aware that immunodeficient patients are at a risk of developing multiple hematological malignancies. A careful examination for other hematological malignancies is needed such patients present with atypical symptoms or an atypical clinical course, even after the diagnosis of the first hematological malignancy.

\section{The authors state that they have no Conflict of Interest (COI).}

\section{Acknowledgement}

The authors would like to express their gratitude to Dr. Seiichi Kato and Dr. Shigeo Nakamura for pathological consultation and helpful discussions.

\section{References}

1. Bernatsky S, Clarke AE, Suissa S. Hematologic malignant neoplasms after drug exposure in rheumatoid arthritis. Arch Intern Med 168: 378-381, 2008.

2. Hoshida Y, Xu JX, Fujita S, et al. Lymphoproliferative disorders in rheumatoid arthritis: clinicopathological analysis of 76 cases in relation to methotrexate medication. J Rheumatol 34: 322-331, 2007.

3. Sekiguchi Y, Shimada A, Imai $H$, et al. Epstein-Barr virusnegative, CD5-positive diffuse large B-cell lymphoma developing after treatment with oral tacrolimus for mixed connective tissue disease: a case report and review of the literature. J Clin Exp Hematop 52: 211-218, 2012.

4. Ochi N, Yamane H, Yamagishi T, Monobe Y, Takigawa N. Methotrexate-induced lymphoproliferative disease: Epstein-Barr virus-associated lymphomatoid granulomatosis. J Clin Oncol 31: e348-e350, 2013.

5. Connors W, Griffiths C, Patel J, Belletrutti PJ. Lymphomatoid granulomatosis associated with azathioprine therapy in Crohn disease. BMC Gastroenterol 14: 127, 2014.

6. Yazdi AS, Metzler G, Weyrauch S, et al. Lymphomatoid granulomatosis induced by imatinib-treatment. Arch Dermatol 143: 12221223, 2007.

7. Lee GC, Hong JS, Lee KH, et al. A case of coincident multiple myeloma and non-Hodgkin's lymphoma. Korean J Intern Med 9: 113-115, 1994.

8. Ibarrola de Andres C, Toscano R, Lahuerta JJ, Martinez-Gonzalez MA. Simultaneous occurrence of Hodgkin's disease, nodal Langerhans' cell histiocytosis and multiple myeloma IgA(kappa). Virchows Archiv 434: 259-262, 1999.

9. Niitsu N, Okamoto M, Nakamine H, Hirano M. Clinicopathologic 
correlations of diffuse large B-cell lymphoma in rheumatoid arthritis patients treated with methotrexate. Cancer Sci 101: 1309-1313, 2010.

10. Liang JH, Lu TX, Tian T, et al. Epstein-Barr virus (EBV) DNA in whole blood as a superior prognostic and monitoring factor than EBV-encoded small RNA in situ hybridization in diffuse large Bcell lymphoma. Clin Microbiol Infect 21: 596-602, 2015.

11. Miyamura K, Osada H, Yamauchi T, et al. Single clonal origin of neoplastic B-cells with different immunoglobulin light chains in a patient with Richter's syndrome. Cancer 66: 140-144, 1990.

12. Xu J, Tang Y, Zhao S, et al. Angioimmunoblastic T-cell lymphoma with coexisting plasma cell myeloma: a case report and review of the literature. Tohoku J Exp Med 235: 283-288, 2015.

13. Swerdlow SH, Campo E, Harris NL, et al. WHO Classification of
Tumours of Haematopoietic and Lymphoid Tissues. 4th ed. IARC Press, Lyon, 2008.

14. Jaffe ES, Wilson WH. Lymphomatoid granulomatosis: pathogenesis, pathology and clinical implications. Cancer Surv 30: 233-248, 1997.

15. Katzenstein AL, Carrington CB, Liebow AA. Lymphomatoid granulomatosis: a clinicopathologic study of 152 cases. Cancer $\mathbf{4 3}$ : 360-373, 1979

The Internal Medicine is an Open Access journal distributed under the Creative Commons Attribution-NonCommercial-NoDerivatives 4.0 International License. To view the details of this license, please visit (https://creativecommons.org/licenses/ by-nc-nd/4.0/).

(C) 2019 The Japanese Society of Internal Medicine Intern Med 58: 2845-2849, 2019 